\title{
CONTACT RESISTANCE
}

BY

IRVING B. SMITH

The instrument maker's art while bound by certain conventions, has of necessity to conform to the requirements of science. Consequently there are various fundamentals of design and construction to be understood and embodied in every well conceived scientific instrument.

We are gradually ignoring many of those conventions which have in them no essential reason for existence. We have outdistanced the tailor, who still puts buttons on the backs of our coats, but we cannot, however, afford to consider only ultilitarian purposes in design and construction. There are certain constructions that remain as the hall-mark of good work; constructions that give pleasure to the conscientious maker and satisfaction to the user; constructions that bring the maker and user together with a sympathetic feeling that some things are worth while for their own sake. We still make binding posts with carefully fitted parts although the expense of doing so is not returned in better operation or more useful application. We put our best efforts into producing materials of high finish and designs of pleasing appearance as a matter of pride while knowing that necessities of service do not require such work. These points offer a means of personal expression tending to elevate the instrument maker's art to an altruistic plane. We hope they will endure.

But, as stated before, there are certain fundamentals, dictated by science and good engineering, that we must always observe if we would produce apparatus of true worth. Knowledge of these requirements should be the common property of all instrument makers and it should be the duty of each of us to aid in the dissemination of this information. These prefatory remarks are a sufficient apology for offering, in the following pages, a rather fragmentary report on the subject of this paper namely, Contact 
Resistance. Contacts between similar or dissimilar electrically conducting materials enter so largely into the construction of instruments as to warrant their careful consideration. The writer has not had sufficient experience to present the subject with the completeness that it warrants but he believes that there is contained herein a considerable amount of data that will be of value to those who design or construct electrical measuring instruments.

In many cases the method of measurement employed is such that the actual resistance values of one or more contacts enter as an error in the measured result. Consider, for example, the measurement of resistance by comparison with known resistances employing the method of Wheatstone's Bridge. This process of

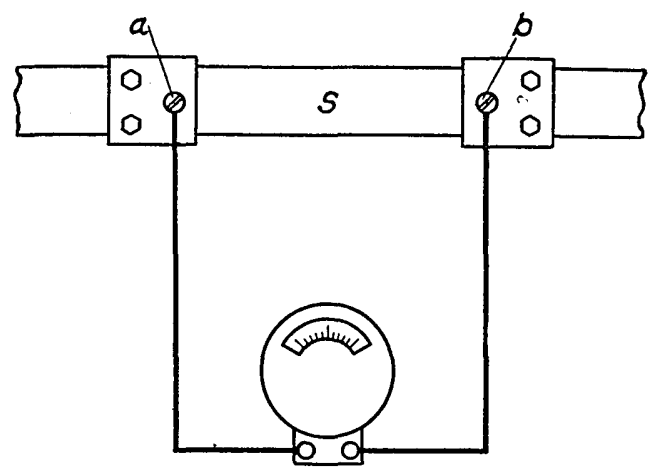

FIG. 1

Millivoltmeter and shunt for measuring heavy currents. Leads are clamped to shunt potential terminals and their resistance and also the contact resistances where attached to the shunt enter into the calibration of the meter.

comparison demands the adjustment of the standard resistances to equality with the unknown or to some determinate ratio. This adjustment may be made with plugs or switches and the result is affected by the actual contact resistances or by changes in contact resistance.

In the measurement of current, by noting the potential drop across a fixed low resistance carrying the current to be measured, errors may arise from contact resistance at the potential points or may be occasioned by a change in current distribution arising from varying resistance at the shunt terminals. For example, a millivoltmeter connected as indicated in Fig. 1 to a shunt $\mathrm{S}$ by 
means of leads attached to terminal screws $a$ and $b$ will have its calibration affected if the contact resistances at $a$ and $b$ vary. Taking the millivoltmeter resistance including leads as $2 \mathrm{ohms}$, an increase of $.001 \Omega$ at each contact point $a$ and $b$ would produce an error in calibration of $1 / 10$ of $1 \%$.

On the other hand there are methods of measurement wherein the actual contact resistance does not cause a direct error. Take, as an instance, the measurement of a potential difference with a potentiometer. Here, the contact elements consisting of switches, plugs or sliders may have a considerable contact resistance without producing an appreciable error in the measurement since the variable contacts may be so arranged as to enter into the galvano-

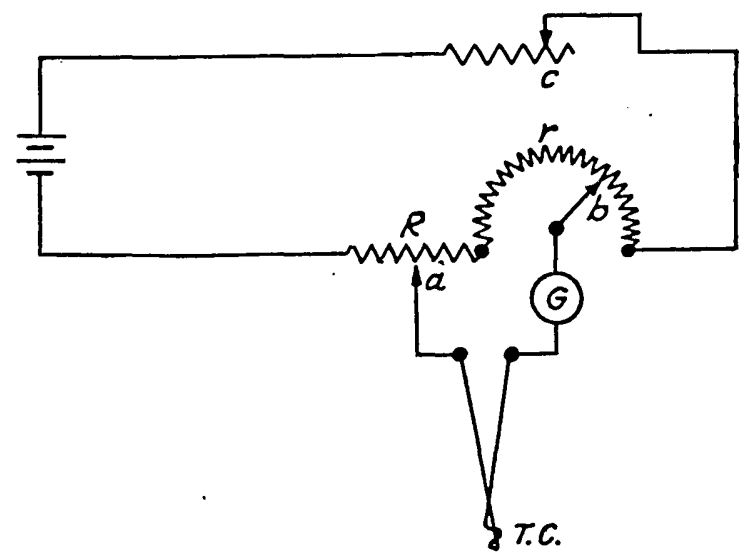

FIG. 2

Simple potentiometer circuit. Standard cell has been omitted. Contacts at $a$ and $b$ are in the galvanometer circuit and only introduce an error to the extent that they lessen the deflection of the galvanometer for a small unbalance.

meter circuit and not into the potentiometer circuit proper. The effect of this arrangement may be to reduce somewhat the sensitivity of the indicator but not the accuracy of the measurement. Referring to Fig. 2, which is a diagram of a simple potentiometer circuit such as one frequently employs for measuring temperature, the thermocouple T. C. is in series with the galvanometer $\mathrm{G}$ and the two sliding contacts $a$ and $b$ are adjusted until the galvanometer indicates no current through its circuit. Since the reading is taken at the time of no current through the contacts $a$ and $b$, it is 
not material whether these contacts vary in resistance excepting that if they increase too much in resistance they will reduce the sensitivity of the instrument and hence the precision of setting. For example, with a thermocouple circuit resistance of $40 \mathrm{ohms}$, an increase in resistance of $5 \Omega$ at each point $a$ and $b$ will reduce the sensitivity $25 \%$. If, therefore, the instrument normally was sufficiently sensitive so that a variation of $.01^{\circ} \mathrm{C}$. was noticeable, it will now be sensitive only to $.0125^{\circ} \mathrm{C}$. It is evident from this that the contact variation impairs the measurement relatively but a small amount. In other cases the effect of contact resistance may be solely a mechanical one and its elimination or lessening may be demanded because of the chance of causing mechanical damage. Bus bars with insufficient area of contact, where clamped together, or where attached to other electrical apparatus, may occasion the production of an amount of heat at the junction great enough to exceed a safe operating temperature. It is evident from this brief discussion that many elements enter into the design and construction of contacts.

The writer, in line with his work for the company with which he is connected, has compiled a data book devoted to the subject of contact resistances. These data have served a useful purpose in the design of electrical measuring instruments. While it is recognized that the notes are fragmentary and the subject by no means exhaustively treated, it is hoped that a brief review of the more important items in this record may be of value to readers of this journal in presenting at least approximate values for the contact resistances usually encountered.

For convenience of reference, contacts have been classified under eight heads. This is arbitrary and more or less artificial since the various types of contact cannot be rigidly separated into classes, each possessing its own peculiar physical characteristics. For example, knife switches partake of the nature of clamped contacts when closed and sliding contacts when being opened and closed. As an approximate guide, resistance values have been set opposite various types of contact, in the following classification, to enable one to form a judgment of the probable value of the resistance to be expected. 


\section{CLASSIFICATION OF CONTACTS}

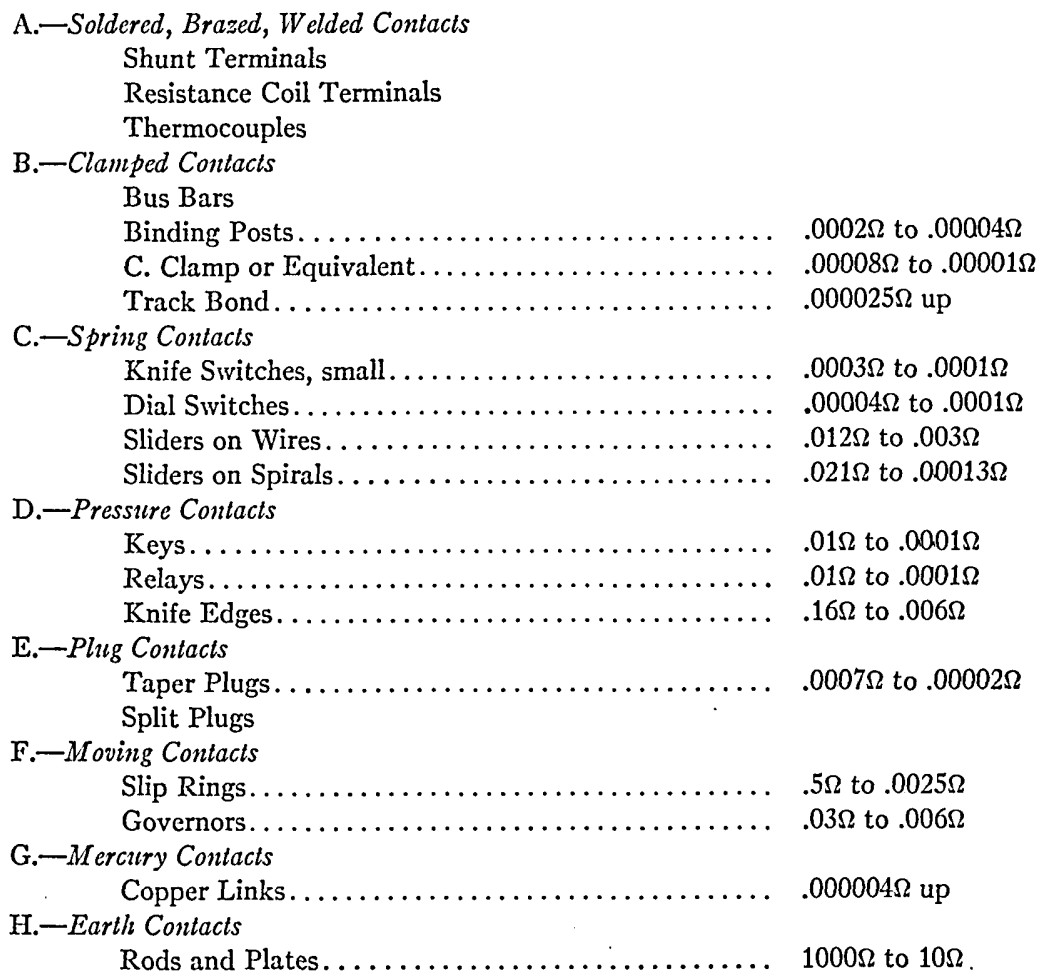

Ludwig Binder (Elekn. Masch. Sept., 1912) in testing between steel balls or between a steel ball and plates of copper, lead or carbon concludes that the nature of resistance at the point of contact is not that of an air or liquid film if the contacts are dry but a true ohmic resistance due to there being but a few points in actual contact. In many practical cases, however, the contact resistance arises from contamination of the surfaces in contact due to chemical action or the presence of foreign substances such as grease or dirt. This phase of the subject, however, we are not considering in the following pages.

In making the following measurements either a fall of potential method was employed or else a Kelvin Bridge method. When employing the first method the connections were as shown in Fig. 3 where the current was supplied by the battery B and measured 
by the ammeter $\mathrm{A}$. Potential points $\mathrm{C}$ and $\mathrm{D}$ were provided by drilling a clearance hole to the point indicated and soldering in insulated wires in such a manner that they were in contact only at their ends. 'The current was usually less than an ampere and the galvanometer $\mathrm{G}$ was calibrated to read microvolts. The battery current was reversed to eliminate Peltier effect. The resistance of the conductors between the potential points could in general be neglected. For example, when measuring the contact resistance between a brass block 1 inch in diameter and a brass plate there was less than $3 / 16$ inch of length of brass between the potential points. Its resistance value, of about $.0000008 \Omega$ was neglected.

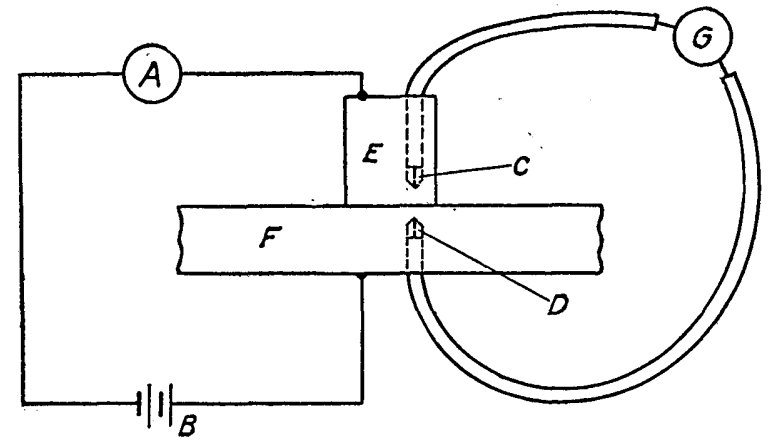

FIG. 3

The contact resistance between brass block $C$ and brass plate $D$ is determined by measuring the current through the contact by means of the ammeter $A$ and the potential drop across the contact by galvanometer $G$. The block $C$ was of round brass $2.5 \mathrm{~cm}$ in diameter milled off square and polished with emery cloth. The brass plate was commercial rolled plate with face polished with emery cloth.

In the determination of the contact resistance of switches and binding posts a similar method was employed. In measurements on binding posts the potential points were arranged as indicated in Fig. 4. The potential wires were of silk insulated copper No. 32 B. \&. S. Gage and the clearance holes small in order to change the current flow lines as little as possible. It will be noted that in this method the contact resistance measured is really that of two resistances in series whereas in actual use the two resistances are in parallel. It is assumed that a fair measure of contact resistance will be gotten by dividing the measured value by two rather than by four. 
In the determination of the contact resistance of plugs the Kelvin Bridge method was used. The connections were in accordance with Fig. 5 where holes were drilled in the brass blocks at $\mathrm{C}$ and $\mathrm{D}$ to such a depth that the bottoms were within $1 \mathrm{~mm}$ of the

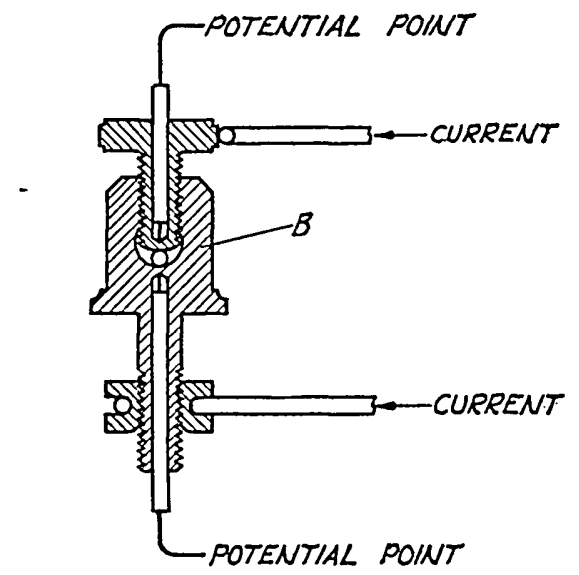

FIG. 4

In measuring the contact resistance of binding posts the current passes through the screw and wire clamped under the post, thence through the body of the post. Potential points are placed, one near the bottom of the screw and one near the bottom of the hole in the body of the post. It is assumed that the contact resistance so measured is double the value to be found in the normal use of the post.

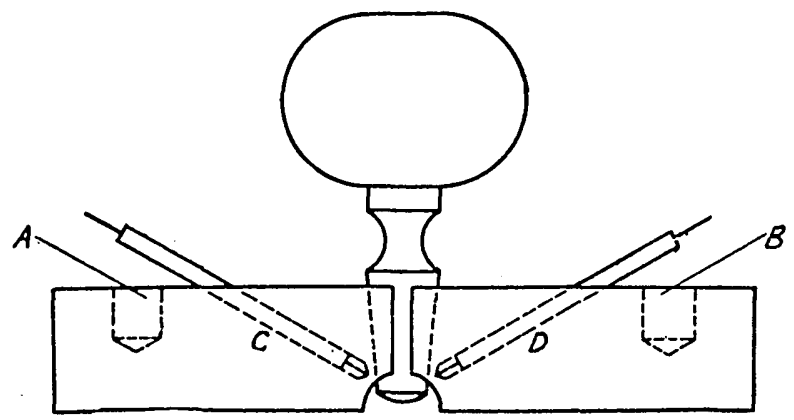

FIG. 5

In measuring plug resistances the potential points are brought close to the plug surface at $C$ and $D$. The current terminals are at $A$ and $B$.

surface of the plug. A very small amount of mercury was placed in each hole and insulated copper wires made contact only at their ends with the mercury. These served as potential points. The current entered the mercury cup at $A$ and left at B. The measure- 
ments were generally taken with a small current since most of the apparatus was for use in electrical measurements where the current values were practically always small. Variations in the measuring current, however, from $1 / 2$ to 3 amperes produced only a small change in the contact resistance measured. For example, in measuring the contact resistance of a phosphor bronze laminated dial switch, the measuring current was varied from 1 ampere to 2.5 amperes resulting in a change in contact from .000175 to $.000184 \mathrm{ohms}$.

It was concluded from this and similar measurements that for the purposes of this investigation the variation of resistance with the current could be neglected since the current in actual service was in general small.

\section{A. SOLDERED, BRAZED, WELDED CONTACTS}

Pieces of round brass and copper rod were squared on the milling machine and joined butt on with commercial soft solder. The solder itself $2.5 \mathrm{~mm}$ in diameter had a resistance of $.0003 \omega$ per $\mathrm{cm}$ of length and as an element in the joint resistance of two quarter inch rods would be less than .8 microhm. Knife edge potential points at a fixed distance apart, were placed first so as to span a section of the rod not including a joint and then so as to include a joint. For two $6 \mathrm{~mm}$ diameter brass rods the joint resistance measured $.000003 \Omega$. For two $3 \mathrm{~mm}$ diameter copper rods the joint resistance measured $.000004 \Omega$. These measurements should have been repeatedly checked but time prevented. They will, however, serve to give one an idea of the order of magnitude of such resistance.

\section{B. CLAMPED CONTACTS}

To study the effect of pressure on clamped contacts, a number of brass blocks were squared off carefully and pressed against a smooth brass plate. The pressure was varied by means of weights and the area of apparent surface contact was varied by employing blocks of different diameter. The actual areas of opposed surfaces do not, of course, determine the area of the contacting parts since it is impossible so to machine the surfaces as to insure contact at all points. It is, therefore, difficult to determine the true 
relations between contact resistance, area and pressure. However, most curves have the form shown in Fig. 6 where the resistance is comparatively high for light pressures and bears a nearly linear relation to the pressure until some value is reached where subsequent increase in pressure does not lower the resistance greatly. Curve A was obtained with surfaces that were polished just before testing. Curve $B$ was for the same surfaces exposed to the atmosphere over night. The materials in contact were respectively a large brass plate and a brass block $2.5 \mathrm{~cm}$ in diameter. This in-

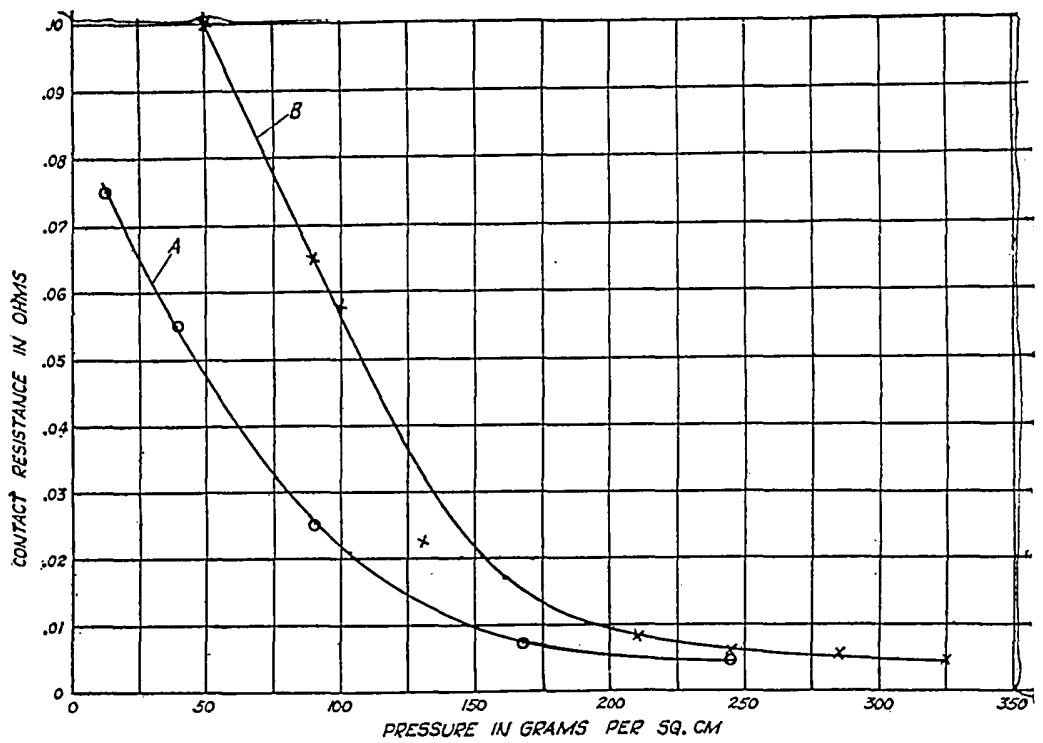

Fig. 6

These curves were the results of tests on the metal blocks shown in Fig. 3. The current was held steady at a known value and the fall of potential measured by a galvanometer. The blocks were both of brass.

dicates that at a pressure of about 200 grams per sq. $\mathrm{cm}$ of apparent contact surface the contact resistance reduces to $.005 \Omega$. Additional experiments with more accurately finished contact surfaces resulted in reducing the contact resistance to $.00005 \Omega$ for a pressure of 800 grams per sq. $\mathrm{cm}$. A considerable variation in resistance was found for different tests. These variations could in general be eliminated by moving the contacting block sidewise while the pressure was on. This adds weight to the usual recom- 
mendation that before finally setting up a clamped contact one of the elements should be driven sidewise slightly.

Binding Posts.-Binding posts are a form of clamped contact used perhaps more often than any other and more frequently employed where their actual contact resistance enters as an error in the measured quantity. If the contacting surfaces are clean, binding posts, when well screwed down, show a resistance usually much lower than the copper leads used to connect them in circuit. For example, two copper leads each of No. 8 B. \& S. wire and each two feet long would offer a resistance of about $.0025 \Omega$. A pair of binding posts suitable for No. 8 wire, if clean and well clamped, would present a resistance of about $.00008 \Omega$. The danger in the use of binding posts resides of course in the fact that they may under various circumstances offer a fairly high resistance. If they are small or not firmly screwed up or dirty a materially higher resistance value results.

Running over all the values given in the writer's data book for large binding posts, small ones, posts with holes for the reception of either wire or flat stock and considering copper, brass, and nickel plated posts, the general conclusion may be reached that the contact resistance varies from $.0008 \Omega$ for dirty and oxidized parts clamped together tightly to $.00004 \Omega$ for clean surfaces firmly clamped. The figures of course vary considerably but assuming that reasonable care is taken to clean and clamp the wire or terminal into a binding post there should be no difficulty in keeping the contact resistance always below.0002 $\Omega$. In exceptional cases where heavy clamps are used instead of binding posts the contact resistance can be kept below $.00001 \Omega$. Flat grip binding posts particularly if not kept clean offer a higher resistance than the type of post where the connecting wire enters a hole and is clamped with the end of the binding post screw. The latter type of post tends to clean the contact elements as the screw is turned down. Brass binding posts offer a less resistance when clean than nickel plated ones but if not kept clean the nickel plated ones show a lower resistance. 


\section{SPRING CONTACTS}

Under spring contacts we shall consider knife switches, dial switches, slider contacts, commercial connectors and generally, contacts whose elements are held together by spring pressure.

There is prevalent a belief that a switch, one element of which has many leaves or laminations is sure to have a low contact resistance. But such a conclusion is not warranted unless the individual laminations are so far independent as to permit each one to operate without interference from the rest. As a rule, when a switch has a large number of leaves, mechanical considerations require that they shall be so close together as to either touch each other or offer small crevices for the lodgment of dirt, particles of lint, etc. These are gradually worked up into the brush until finally the brush operates as though it were one solid structure; and moreover, the material forced between the laminations has lifted some of them so that the contacting face is no longer in proper bearing. On the other hand a laminated brush possessing say two or three or at most a half dozen leaves offers no serious mechanical difficulty in the way of proper spacing of the leaves with the result that such a brush shows consistently low in contact resistance, assuming of course that it is properly constructed, being more likely to improve itself with age because of the wearing in and more intimate fitting of the various parts. We have had occasion to measure the brush resistance of switches possessing a large number of laminations packed so closely as to prevent individual leaves from having really independent motion and have found the resistance to be higher than that of brushes of much simpler construction, and moreover, they tend to increase their contact resistance as stated above because of their picking up and holding lint, copper dust, dirt etc. When new, a switch of this type is low in resistance but as it wears it is likely to show a higher resistance. Another point for consideration is that when the laminations are closely packed and are in considerable numbers the brush has practically no elastic recovery. As a result the smallest wear eases up on the brush contact pressure and adds to the contact resistance. It has also been noticed that, when a brush of this type is mounted on a hard rubber plate, the continued 

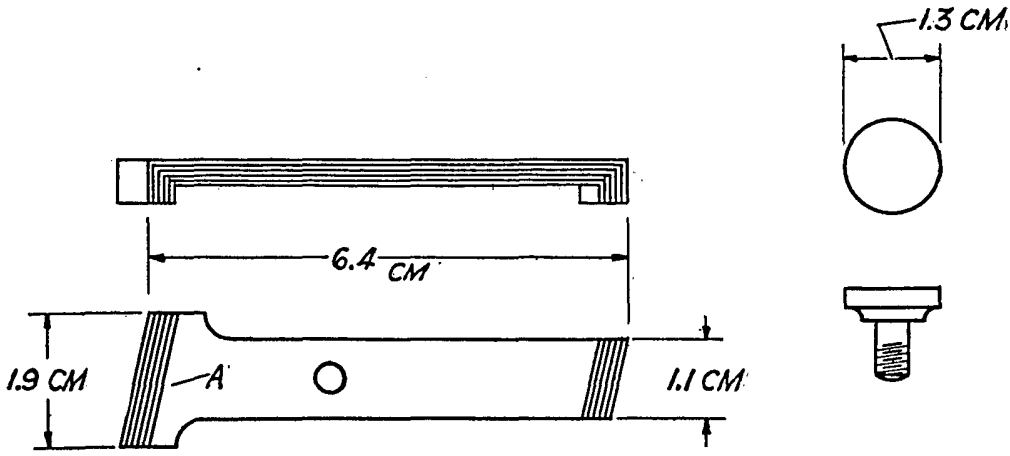

FIG. 7

Brush No. 102

Six phosphor bronze laminations $.4 \mathrm{~mm}$ thick

For contact resistance at end $A$ see Fig. 14, curve $A$. With this brush there was employed contact stud No. 87, Fig. 12. Stud was of brass. Normal pressure is such that the contact resistance is about $.00023 \Omega$ at each end of brush. The same combination was tested when the stud was nickel plated. See Fig. 14, curve $B$. Normal resistance about $.00045 \Omega$ at each end of brush.

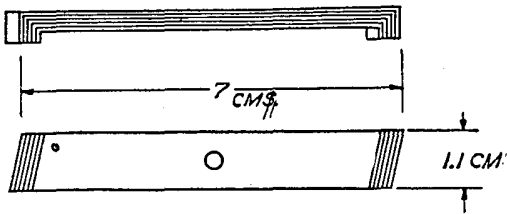

FIG. 8

Brush No. 101

Six phosphor bronze laminations $.4 \mathrm{~mm}$ thick

For contact resistance at one end, see Fig. 14, curve $C$. With this brush there was employed contact stud No. 86. Nickel plated. See Fig. 13. Normal pressure is such that the contact resistance is about $.00045 \Omega$ at_each end of brush.

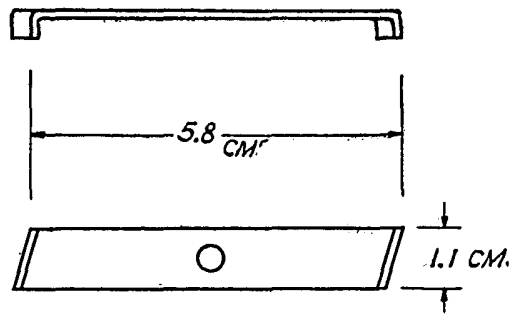

FIG. 9

Brush No. 100

Two phosphor bronze laminations $.8 \mathrm{~mm}$ thick

For contact resistance at one end, see Fig. 14, curve $F$. With this brush there was employed contact stud No. 87 nickel plated, Fig. 12. Normal pressure is such that the contact resistance is about $.0008 \Omega$ at each end of brush. 
pressure has caused the rubber to give sufficiently to materially increase the contact resistance.

The contact resistances of various types of switches are indicated under Fig. 7 to 13 inclusive, together with curves showing the relation between pressure and resistance. Referring to Fig. 14 the conditions under which curves $\mathrm{A}, \mathrm{B}, \mathrm{C}, \mathrm{D}, \mathrm{F}$ and $\mathrm{H}$ were gotten are indicated under Figs. 7 to 13. Curve $\mathrm{E}$ was for the same brush

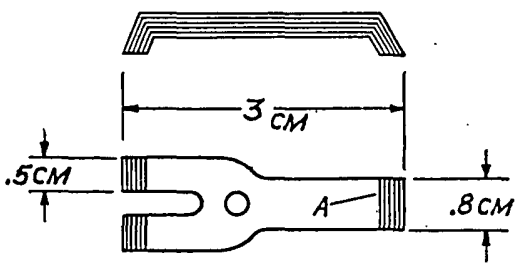

FIG. 10

Brush No.B

Nine phosphor bronze laminations $.4 \mathrm{~mm}$ thick not spaced apart

For contact resistance at end $A$ see Fig. 14, curve $B$. With this brush there was employed stud No. 86, Fig. 13. Stud of brass not nickel plated. Normal pressure of about $4 \mathrm{~kg}$ per sq. cm results in a contact resistance of about $.0003 \Omega$.

Brush No. S

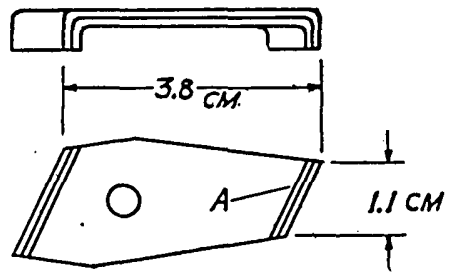

FIG. 11

Three phosphor bronze laminations $.4 \mathrm{~mm}$ thick

For contact resistance at end $A$ see Fig. 14, curve $D$. With this brush was employed contact stud No. 87 , Fig. 12. Stud was of brass. Normal pressure such that contact resistance at end $A$ is about $.0002 \Omega$.

and stud as shown in Fig. 7 excepting that the stud was of bare copper. Curve $\mathrm{G}$ was for the same brush and stud also as indicated in Fig. 7 but the stud was of bare brass. It differed from the conditions of curve $\mathrm{A}$ only in the fact that it was new whereas the brush and stud corresponding to curve $\mathrm{A}$ had been used for some time.

In most instances we are interested in the variation in the contact resistance rather than in its actual value since the cali- 
bration or adjustment of an instrument often takes account of the absolute value of the resistance at contacts but cannot compensate for variations in such resistances. With the switch parts used in determining curve $\mathrm{C}$ the contact resistance for a normal pressure
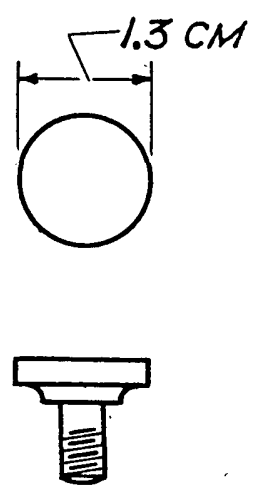

FIg. 12

Type of brass or copper switch stud employed. A potential point was located just under the top surface of stud.
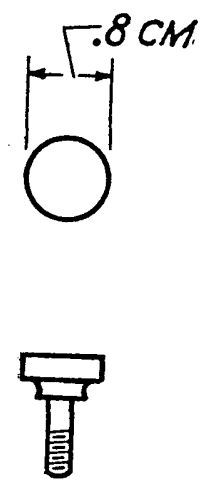

FIG. 13

This stud differed from that shown in Fig. 12 in that it was of smaller diameter.

of $2 \mathrm{~kg}$ was about $.0003 \Omega$ and its variation approximately $.00006 \Omega$. Brush pressures normally run from 1.5 to $3 \mathrm{~kg}$. Contact resistances, therefore, may be expected to range from $.00045 \vec{\Omega}$ to $.00015 \Omega$.

KNIFE SwITCHES-These are frequently employed in small sizes on measuring instruments. A 15-ampere commercial knife switch old and not used for some time varied in resistance from $.0001 \Omega$ to $.0003 \Omega$. A similar but new one varied from .00007 to $.0001 \Omega$. A third familiar form whose clips were bent back on themselves varied 
from $.00019 \Omega$ to $.00024 \Omega$ this high resistance being due to the fact that the doubled length of clip prevented securing an adequate pressure against the knife blade.

Small Connectors. - The usual type of connector used in automobile head lights had a contact resistance varying from .003 to $.007 \Omega$.

FAHNSTOCK Clip.-When clamping a piece of nickel plated copper wire, No. 12 B. \& S. Gage, Fahnstock clips varied in contact resistance from $.00005 \Omega$ to $.0005 \Omega$ depending upon the material of the clip and its size.

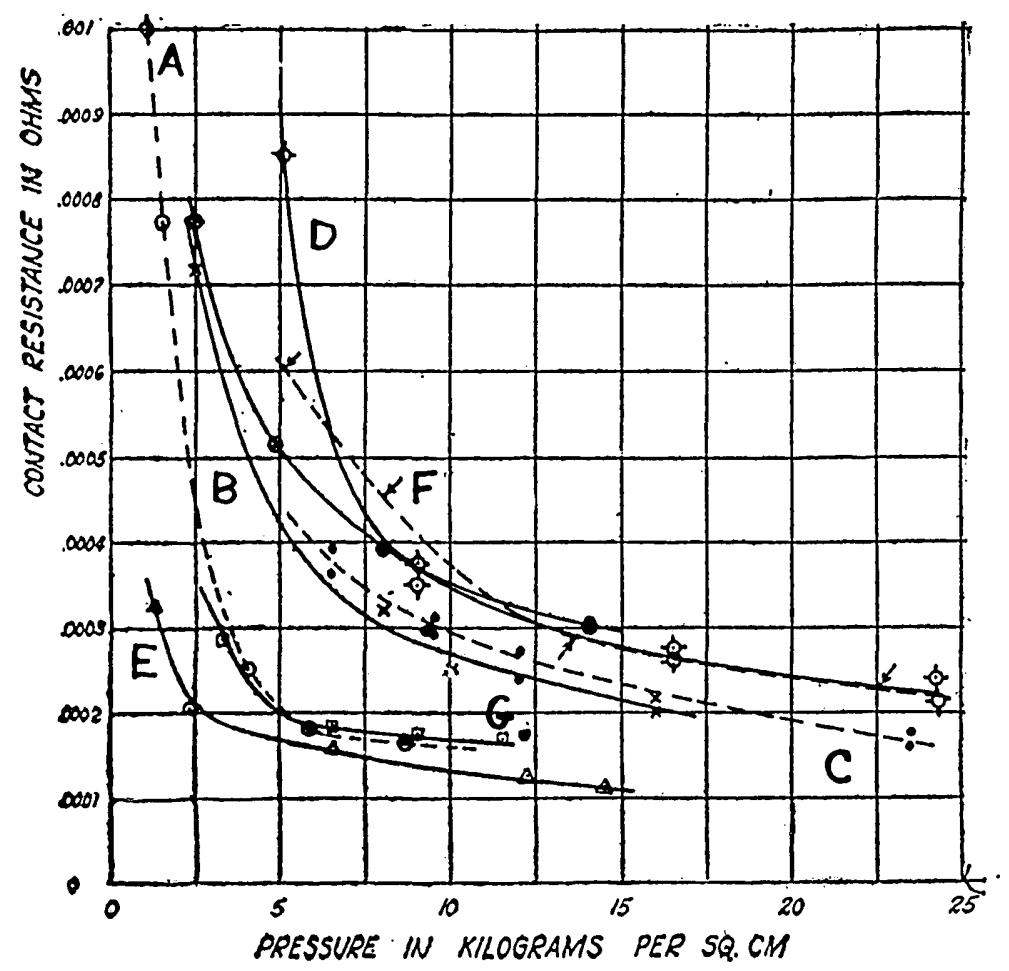

FIG. 14

The above curves were obtained with switches of various types. The previous Figs. 7 to 13 inclusive give the principal dimensions of these switches and reference is made by letter to the corresponding curve in Fig. 14.

Slider Contacts.-For many purposes a knife edge or small surface is used to make contact against a slide wire or a wire helix and it is held in contact with a nearly constant spring pressure. Motion occurs between the slider and the wire but the resistance of the contact enters for consideration only when the slider is at rest. As a rule, devices of this nature are employed in such a manner that the effects of contact resistance do not enter into the measurement excepting as they indirectly influence it by affecting the sensitivity of the indicating device. For this reason a fairly high contact resistance is often permissible. 
Various materials have been used for the slider and for the slide wire or spiral. The following conclusions from a large number of tests were reached.

1. A Copper gauze slider tends to clean the wire upon which it slides.

2. A Steel slider is more variable than a gauze one but in some instances is to be preferred because of its better wearing qualities.

3. A Silver slider is best from the standpoint of low resistance and constancy but wears rapidly.

To review even briefly the numerous tests that have been made on slide wires and spirals would require too much space. A few figures, therefore, will be furnished to give one an idea of the range of contact resistance to be expected.

1. Steel Slider on Straight Therlo Wire.-Pressure 37 grams. Contact resistance varied from $.006 \Omega$ to $.05 \Omega$ when dirty and from $.006 \Omega$ to $.012 \Omega$ when cleaned by rubbing off with a piece of cloth.

2. The same combination but allowed to become very dirty by standing in a furnace room for five weeks. Without removing the dirt the contact resistance reached $.3 \Omega$. When wiped off the highest value was $.03 \Omega$ and a more careful cleaning reduced the resistance to $.008 \Omega$ maximum.

3. A similar test to No. 2 for a steel slider on manganin wire showed contact resistances as high as $.12 \Omega$ after wiping clean indicating that Therlo is better as far as this characteristic goes than manganin.

4. Silver Slider on Therlo Wire.-When new and clean the contact resistance varied from $.0017 \Omega$ to $.003 \Omega$ and when dirty the variation was from $.0025 \Omega$ to $.008 \Omega$.

5. Copper Gauze Slider on Therlo Wire.-When new and clean the contact resistance varied from $.002 \Omega$ to $.003 \Omega$ and when dirty the variation was from .003 to $.5 \Omega$. This excessive resistance was due to dirt accumulating in the meshes of the copper gauze. After filling the gauze the variation was from .002 to $.003 \Omega$ as it was when new.

6. Silver Slider on Silver Wire.-When new the contact resistance varied from $.00013 \Omega$ to $.0002 \Omega$ and while dirty it varied from $.00013 \Omega$ to $.00022 \Omega$. After about two months standing the resistance varied from $.0003 \Omega$ to $.0009 \Omega$.

Various other combinations were tried out but those cited above give one an idea of the contact resistance to be expected. In the above cases the slider pressure against the slide wire was about 40 grams. It is an interesting fact that if the slide wire is in the form of a closely wound helix the cleaning action of the slider as it moves over the wire from turn to turn is better. than if the slide wire were straight. 
D. PRESSURE CONTACTS

Under this rather vague title we include contacts which are held together by finger pressure or by electromagnetic devices, such for example as keys and relays. We will refer only briefly

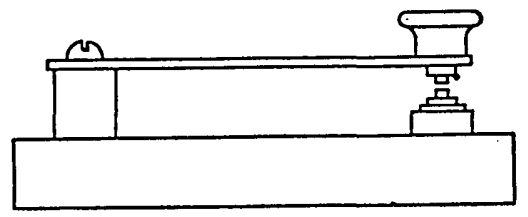

FIG. 15

This conventional type of key had silver contacts about $2 \mathrm{~mm}$ in diameter. The potential points were determined by drilling very fine holes in the silver contacts near their interface and peening copper wires $.1 \mathrm{~mm}$ diameter in the holes.

to these. In an old standard type of key, Fig. 15, with silver contacts the following contact resistances were observed:

Before cleaning:

$\begin{array}{cc}\text { Pressure } & \text { Resistance } \\ \mathrm{kg} & \Omega \\ .1 & .085 \\ .45 & .0038 \\ 3.20 & .0006\end{array}$

After cleaning (with fine emery):

$\begin{array}{cc}\text { Pressure } & \text { Resistance } \\ \mathrm{kg} & \Omega \\ .1 & .00095 \\ .45 & .00037 \\ 1.80 & .00023 \\ 3.20 & .0001\end{array}$

A telephone jack showed a contact resistance of $.0025 \Omega$ for one spring and $.0075 \Omega$ for another. These contacts were relatively high because the contacts are so constructed that the contact surfaces come together without sliding and therefore without any cleaning action.

Another interesting test was on a galvanometer used as a relay to close an electric circuit when the galvanometer pointer deflected against a fixed contact. Both contact elements were of platinum. The results are best shown by the curve in Fig. 16. It 
might be well to add that the values shown in the curve were for clean contacts and they would be materially affected by even a small amount of dirt.

\section{E. PLUG CONTACTS}

The resistance of a plug contact varies, of course, with its condition, its construction and the degree of pressure exerted in seating the plug. Fig. 17 gives one an idea of the contact resistance of a

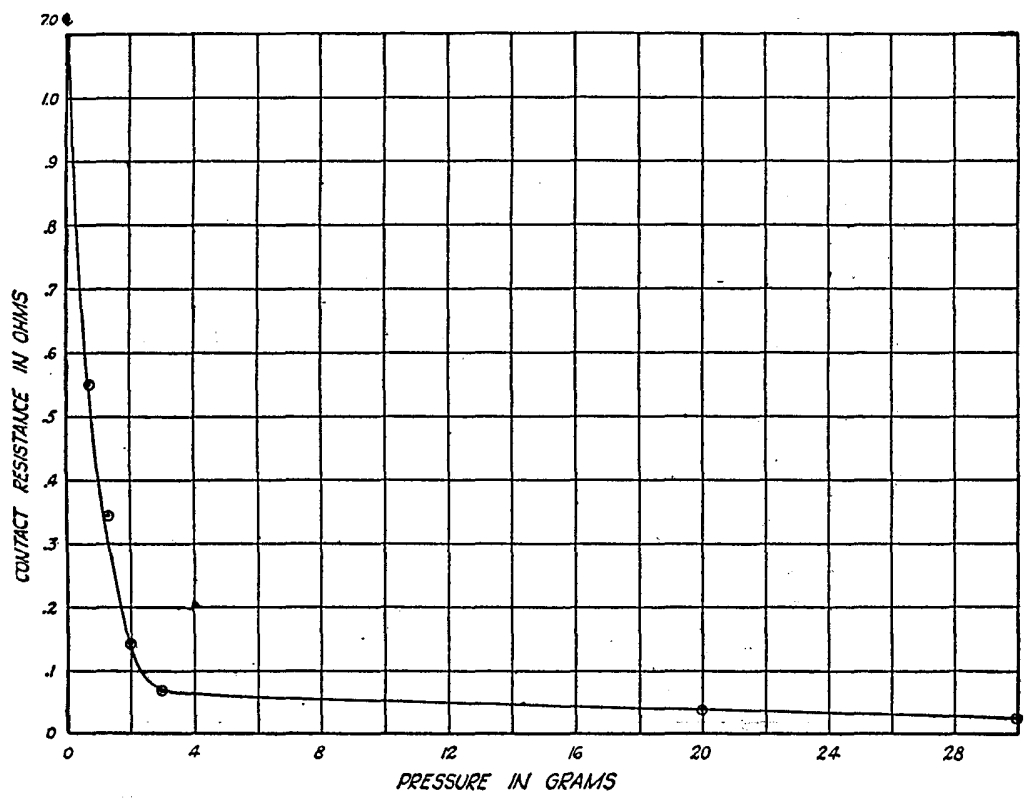

FIG. 16

Contact resistance between two crossed platinum wires of No. 22 B. \& S. Gage. One wire was a pointer on a galvanometer used as a relay and the other wire acted as a stop to close a circuit when pointer was sufficiently deflected.

large taper plug in good condition set in with various degrees of pressure. For this test a plug and blocks were used of the dimensions indicated in Fig. 18. The potential points and current points were as shown in Fig. 5. While the pressure was applied and before each reading, the plug was given a slight twist. There is an appreciable reduction in contact resistance up to a pressure of 8500 grams after which the gain is small. At that pressure the resistance is about .000025 . This is really a measure of two contacts 
in series since the current path was from block $A$ to block $B$ in Fig. 5. Somewhat lower values than those shown on the curve of Fig. 17 were gotten in other tests but a fair allowance for the

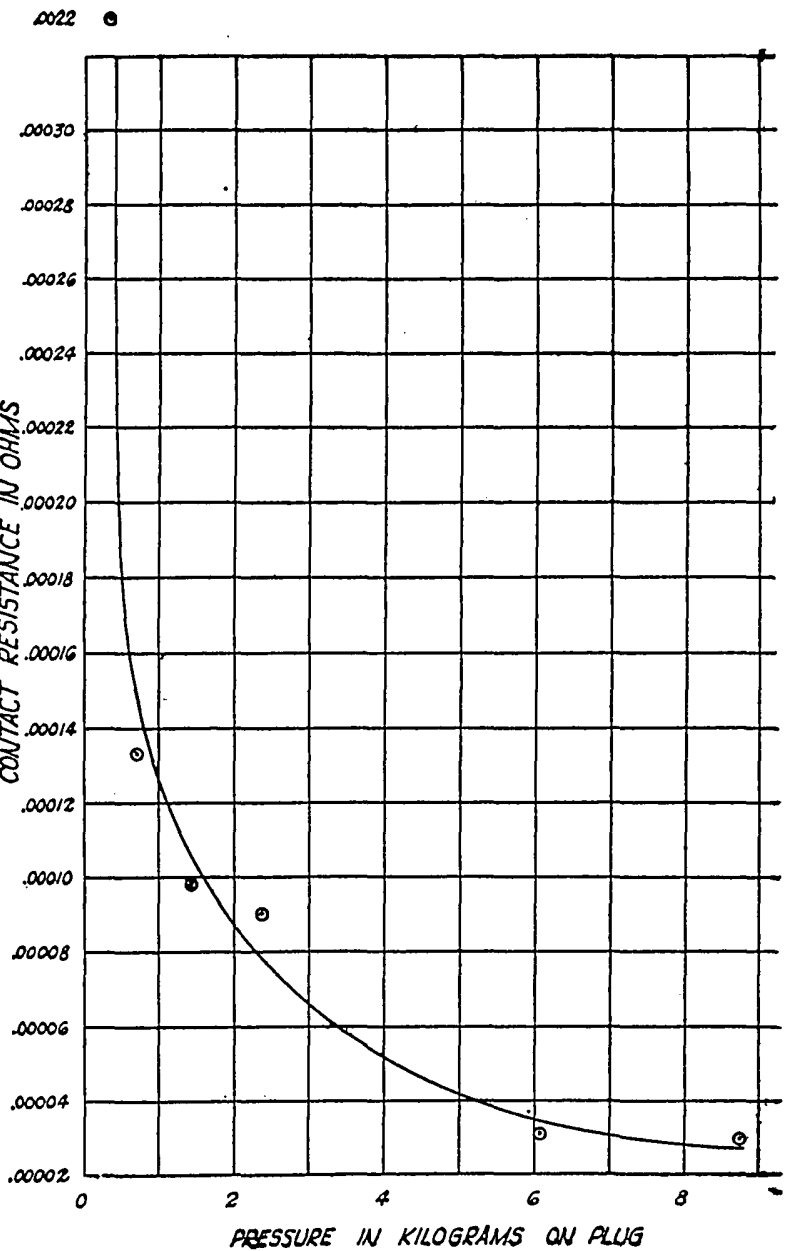

FIG. 17

Contact resistance between a standard taper plug and its block. In making the measurements plotted here the potential points were so located that two contacts were in series. See Fig. 5.

contact resistance of a large plug well fitting and firmly seated with a twisting motion is about .000025 . To give one an idea of the resistance variation under these conditions the plug was taken 
out and reseated before each instrument. Of thirteen measurements the mean value was $.0000212 \Omega$ with variations $\pm .0000007 \Omega$.

By way of comparison a similar plug was measured both when dirty and after reaming the hole and cleaning the plug. When dirty the resistance varied from $.0004 \Omega$ to $.0008 \Omega$. After cleaning it varied from $.0000205 \Omega$ to $.0000251 \Omega$. This variation of \pm .000005 is more likely to be encountered than that given above. From this and other tests it appears reasonable to assume the resistance of a good plug contact to be about $.000025 \Omega \pm .000005 \Omega$. A plug of the above size is normally set with a pressure around $12-13 \mathrm{~kg}$. For a smaller plug and for blocks not capable of withstanding such pressure, the contact resistance may be about $.00005 \Omega \pm$ $.00001 \Omega$.

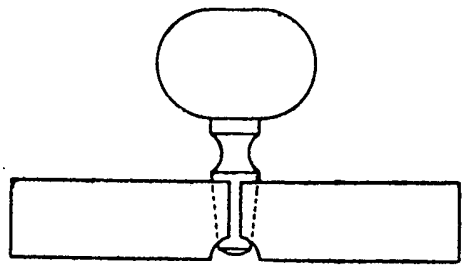

FIG. 18

Standard taper plug used in making measurements plotted in Fig. 17. The drawing represents actual size of plug and blocks. The plug taper was $1 \mathrm{~mm}$ increase in the diameter per cm length.

F. MOVING CONTACTS

From the standpoint of the instrument maker continuously moving contacts are of interest when carrying small currents and his interest rests more upon the constancy of resistance than the current carrying capacity. An illustration related to the instrument maker's art is that of slip rings and brushes used on rotating sockets in photometric measurements. The following is a test on such a piece of apparatus.

Contact resistance of slip ring

Slip rings brass.

Brushes copper gauze.

Pressure 150 grams.

Standing .0019 .

$180 \mathrm{rpm} . .026 \Omega$.

$450 \mathrm{rpm} . .035$ to $.05 \Omega$. 
A phosphor bronze leaf brush of 6 leaves was substituted.

Pressure 200 grams.

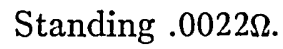

$180 \mathrm{rpm} . .0025 \Omega$.

$350 \mathrm{rpm} . .0029 \Omega$.

The contact resistance of phosphor bronze brushes on a copper slip ring was found to be $.0045 \pm .001 \Omega$ when rotating at $80 \mathrm{rpm}$. and $.0004 \Omega$ when standing. In this case the brush consisted of 3 leaves of $.4 \mathrm{~mm}$ phosphor bronze $12 \mathrm{~mm}$ wide having a total brush pressure of $2 \mathrm{~kg}$.

Contact resistance of bearing

A half inch cold drawn steel shaft running in a brass bearing $13 / 4$ inches long at $2000 \mathrm{rpm}$. showed a contact resistance when oiled of $.140 \Omega$ not oiled of $.05 \Omega$.

The bearing load was about $2.25 \mathrm{~kg}$.

\section{G. MERCURY CONTACTS}

In Reprint (Sci. Pap.) No. 241 of the Bureau of Standards the subject of mercury contacts is considered. The resistance at the contact of one mercury contact may by proper design and construction be reduced to about $.000004 \Omega$. When in poor conditions, however, owing to an accumulation of dirt a much higher value may result.

\section{H. EARTH CONTACTS}

The conditions may vary to such a degree that it is difficult to fix any limits on the resistance of earth contacts. Values were gotten as low as $10 \Omega$ when using ground plates 12 inches square in well watered earth and as high as $400 \Omega$ when using a half inch bright copper rod.

The LeEds and Northrup Instrument Co., Philadelphía, Pa. 